\title{
Wave-Mixing Analysis for THz-Signals Generation in dc-Biased Semiconductor Optical Devices at Room Temperature
}

\author{
Ramón Maldonado-Basilio, Sylwester Latkowski, Frederic Surre and Pascal Landais \\ Research Institute for Networks and Communications Engineering \\ Dublin City University, Glasnevin, Dublin 9, Ireland \\ Corresponding author: landaisp@eeng.dcu.ie
}

\begin{abstract}
Wave-mixing at $370-\mathrm{GHz}$ in a SOA and FP semiconductor laser is investigated by using a FROG-system. A comparison of the optical time-fluctuations measured at their output stresses the importance of a resonant cavity in $\mathrm{THz}-$ signals generation from semiconductor optical devices.
\end{abstract}

\section{INTRODUCTION}

Techniques for generating THz-waves $(0.3$ to $10 \mathrm{THz})$ are attracting significant interest due to their potential applications in spectroscopy, imaging, sensing, and security. There are a number of approaches to generate $\mathrm{THz}$ radiation [1-2]: free electron lasers, Schottky resonant tunnel diode oscillator arrays, P-type Ge lasers, quantum cascade lasers, photoconductive switches, and photomixers coupled to an antenna. In order to obtain a $\mathrm{THz}$ signal based on these approaches, a variety of challenges have to be overcome. Among the most important ones are the complexity of the structures, necessity of cryogenic cooling temperatures, large electrical power consumption, and limitations on spectral tunability.

A cost-effective approach recently investigated for generation of continuous-wave (cw) THz-signals is based on semiconductor lasers operating at room temperature. Hoffmann et al [3] have demonstrated direct emission at $\mathrm{THz}$ frequencies out of a two-color laser coupled into an external cavity. Similarly, our research team have demonstrated the generation of 370-GHz waves [4] and extended the analysis up to $1.15 \mathrm{THz}$ [5] by using spectrum tailored Fabry-Pérot (FP) semiconductor lasers at room temperature. The $370-\mathrm{GHz}$ signal measurement was carried out both directly and indirectly. In the former case, we use a cryogenically cooled bolometer interfaced to a Fourier Transform - Infra Red (FTIR) spectrometer with IR filtering schemes to remove the laser radiation. In the latter one, we use a frequency resolved optical gating (FROG) technique. Both measurement approaches demonstrated the electromagnetic wave generation within the $\mathrm{THz}$ range by this sort of multimode laser.

In this work, we propose a further experimental study on THz-signals generation by semiconductor devices. To this end, wave mixing analysis at $370-\mathrm{GHz}$ on a spectrum tailored FP laser and a semiconductor optical amplifier (SOA) is addressed. In the former case, the multimode FP laser described in [4-5] is used. In the latter case, two cw-beams emitted by two independent tunable external cavity lasers (ECL) are injected into a dc-biased SOA. Thus, considering the same beating frequency and therefore the same frequency of the $\mathrm{THz}$ generated signal (i.e. two main-modes frequency separation for laser and ECLs frequency detuning for SOA), a comparison on the performance of wave mixing achieved by these devices is drawn. From these experimental results, an explanation is given for the $\mathrm{THz}$ generation process and the importance of the laser cavity is stressed.

\section{EXPERIMENT}

To start with, the wave mixing analysis is performed on the SOA. It is an InGaAsP bulk active layer device. When dcbiased at $400 \mathrm{~mA}$ and temperature controlled at $25^{\circ} \mathrm{C}$, its maximum fibre-to-fibre small signal gain is $25 \mathrm{~dB}$ at $1555 \mathrm{~nm}$ with a $3 \mathrm{~dB}$ bandwidth of $55 \mathrm{~nm}$. The first step is to prove that the two cw-beams from the ECLs are not modulated by any means before their injection into the SOA. This is achieved by simultaneously injecting the two ECLs into the FROG system, referenced as HDR 150 from Southern Photonics. The wavelength of the injected signals are $\lambda_{1}=1552.7 \mathrm{~nm}$ and $\lambda_{2}=1555.7 \mathrm{~nm}$, each of them with a mean power of $5 \mathrm{dBm}$. The FROG spectrogram of the second harmonic generation associated to each beam appears constant in time.

Secondly, the two cw-lasers are injected into the SOA. Since these beams are within the $3 \mathrm{~dB}$ gain of the amplifier, they experience a similar gain and a strong four-wave mixing (FWM) signal is observed after proper optimization on their state of polarization. Using a $5 \mathrm{~nm}$ tunable band-pass filter centered at $1554.2 \mathrm{~nm}$, the two main wavelengths at the SOA output are selected for simplicity of the analysis and launched into FROG. Time evolution of the second harmonic signals at $\lambda_{1} / 2$ and $\lambda_{2} / 2$ obtained at the SOA output are presented in Fig. 1. The "+" symbols stand for the experimental data and the continuous line is obtained by a sinusoidal fitting function. It is noticeable that the second harmonic signals exhibit a periodic modulation after propagating through the SOA, even though the amplifier is dc-biased and the input signals are in 
$\mathrm{cW}$ regime. Mode beating from FWM produces a modulation of the complex refractive index in the SOA active region. This results in a periodic fluctuation at the beating frequency of 370 $\mathrm{GHz}$ on the amplitude and phase of the injected beams. Indeed, a constant phase difference $\varphi=\pi$ between the two main modes at the SOA output is observed. At such a high beating frequency, the carrier heating process is governing the dynamics on the amplifier.

The second semiconductor device under investigation is a $350 \mu \mathrm{m}$ long multi-quantum well InAlGaAs spectrum tailored FP laser [4-5]. It is dc-biased at $85 \mathrm{~mA}$ and temperature controlled at $25^{\circ} \mathrm{C}$. The optical spectrum presents two main modes separated by $370 \mathrm{GHz}$ with two FWM side-bands clearly visible. As in the case of the SOA, the two main modes are selected and launched into the FROG system. A periodic fluctuation associated with the main modes and a constant phase difference $\varphi=\pi$ between them is exhibited, as shown in Fig. 2.

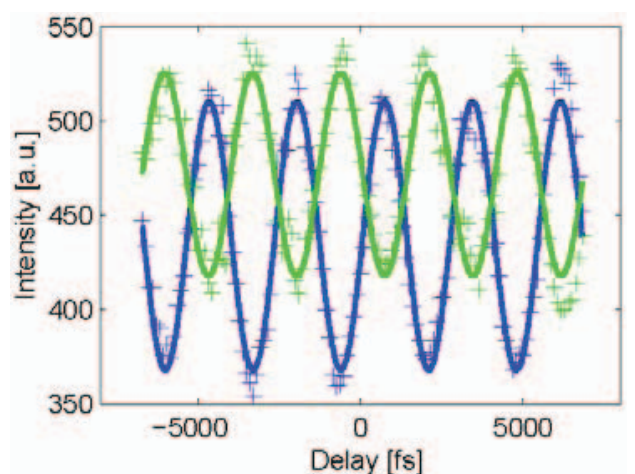

Fig. 1. Time evolution of the main modes $\lambda_{1} / 2$ and $\lambda_{2} / 2$ at the SOA output.

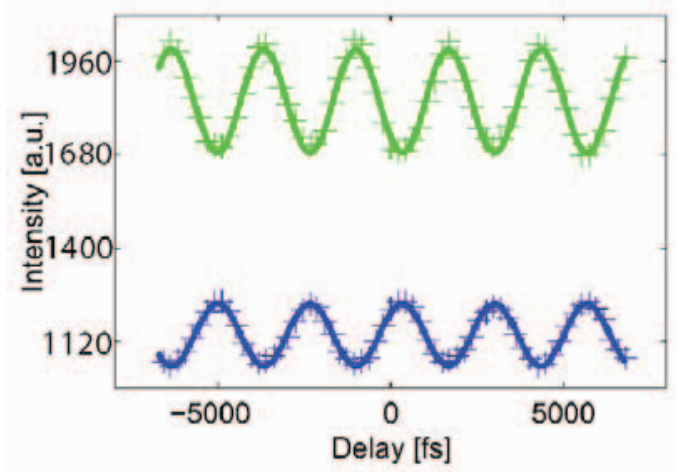

Fig. 2. Time evolution of the main modes $\lambda_{1} / 2$ and $\lambda_{2} / 2$ at the FP laser output.

Comparing the measurements depicted in Figs. 1 and 2, the time fluctuation of the second harmonic signals associated to the main modes at the output of the laser is larger than that of the SOA. It is worth mentioning that the power spectral density of the two main modes measured at the FP laser output is about $-20 \mathrm{dBm}$, whilst the power spectral density associated to each beam at the SOA output is above $+8 \mathrm{dBm}$ for an injected mean power of $-2 \mathrm{dBm}$. The reported values are measured by using an optical spectrum analyzer set at a resolution of $0.05 \mathrm{~nm}$. Moreover, a ratio of $5 \mathrm{~dB}$ between the main beams and the conjugate signal is measured at the SOA output under these experimental conditions, which is an indication of its operation in nonlinear regime. In both FP laser and SOA, the measured signal to noise ratio is well above $40 \mathrm{~dB}$. At these rather different conditions in power levels, it appears that the peak-to-peak amplitude from the periodic modulation undergone by the laser modes is stronger. All these differences suggest the mode beating (and consequently the optically generated signal at $370 \mathrm{GHz}$ ) present in the active layer is more efficient when implemented in the FP laser than in the SOA.

\section{CONCLUSIONS}

Wave-mixing at $370 \mathrm{GHz}$ in a FP laser and SOA has been investigated for its application in all-optical THz-signals generation. In order to achieve an efficient $\mathrm{THz}$ down conversion process, optical devices with active layers of high third order non-linear susceptibility should be used. Additional consideration should be given to weather the devices are FP or traveling-wave amplifiers. In the case of a FP laser, the fourwave mixing mechanism benefits from a better overlapping between all the optical modes selected by the cavity. Therefore, more efficient THz-waves can be generated by mode beating with a FP laser than a semiconductor optical amplifier, even though the SOA concept offers a wide frequency tunability.

\section{REFERENCES}

[1] G. L. Carr, M. C. Martin, W. R. McKinney, K. Jordan, G. R. Neil, and G. P. Williams, "High-power terahertz radiation from relativistic electrons," Nature 420, pp. 153-156, 2002.

[2] M. A. Belkin, F. Capasso, F. Xie, A. Belyanin. M. Fischer, A. Wittmann, and J. Faist, "Room temperature terahertz quantum cascade laser source based on intracavity difference-frequency generation," Appl. Phys. Lett., 92(20), 201101, 2008.

[3] S. Hoffmann, X. Luo, and M. Hofmann, "Bandwidth limitations of twocolour diode lasers for direct terahertz emission," Electron. Lett. 42(12), pp. 696-697, 2006.

[4] S. Latkowski, F. Surre, and P. Landais, "Terahertz wave generation from a dc-biased multimode laser," Appl. Phys. Lett. 92(8), 081109, 2008.

[5] S. Latkowski, F. Surre, R. Maldonado-Basilio, and P. Landais, "Investigation on the origin of terahertz waves generated by dc-biased multimode semiconductor lasers at room temperature," Appl. Phys. Lett. 93(24), 241110, 2008. 